\title{
Perceptual load modulates attentional capture by abrupt onsets
}

\author{
Joshua D. Cosman and Shaun P. Vecera \\ University of Iowa, Iowa City, Iowa
}

\begin{abstract}
The abrupt appearance of a new object captures attention, even when the object is task irrelevant. These findings suggest that abrupt onsets capture attention in a stimulus-driven manner and are not susceptible to top-down influences on attentional control. However, previous studies examining the ability of abrupt onsets to capture attention have used search displays that lacked significant complexity. Because attention is a limited capacity mechanism, it is possible that increasing the complexity, or perceptual load, of the search arrays may modulate capture by abrupt onsets. We used a flanker task to examine the effect of perceptual load on attentional capture by abruptly appearing objects. Subjects searched for a target letter through low-load (set size $=1$ ) and high-load ( Onset flankers affected search in low-load but not high-load displays. This modulation of attentional capture was not caused by generalized slowing when subjects searched through high-load displays; search for a single perceptually degraded target slowed response times but did not affect attentional capture. These findings demonstrate that attentional capture by an abrupt onset is attenuated when people search through high-load scenes.
\end{abstract}

Attention can be voluntarily directed to specific locations or objects in a scene, allowing us to process only the subset of information relevant to our current goals. However, in some cases, information not currently relevant can capture attention. As a result, most accounts of attention postulate that both bottom-up (stimulus-driven) and top-down (goal-directed) factors compete to direct attention within a visual scene. It is generally accepted that attention can be deployed voluntarily, but whether or not attention can be captured in a purely stimulus-driven manner remains an open question (see Folk, Remington, \& Johnston, 1992; Franconeri, Simons, \& Junge, 2004).

Many dynamic stimuli appear to be capable of capturing attention in a strictly stimulus-driven manner (Abrams \& Christ, 2003; Franconeri \& Simons, 2003; Theeuwes, 1991; Yantis \& Jonides, 1984). For example, the abrupt onset of a new object in a visual scene captures attention. Yantis and Jonides (1984) used an irrelevant feature search task to measure capture by abruptly appearing objects. Observers first viewed a placeholder array, which was followed by a search array containing a target letter and several distractors. Most of the letters in the search array were created by the disappearance (i.e., offset) of features from a placeholder, but one letter in the search array appeared at a location not occupied by a placeholder; this item appeared abruptly as a new object whose features had not been present in the placeholder array. The target was no more likely to appear as an onset than as an offset, providing no incentive for giving onsets an attentional priority during search. Despite this, Yantis and Jonides (1984) reported shallower search slopes when the target was an onset, suggesting that the abruptly appearing onset items captured attention in a stimulus-driven manner.

Like onsets, offsets also appear to be capable of capturing attention in a stimulus-driven manner in some situations. For instance, it has been demonstrated that during search, offsets can capture attention when accompanied by a luminance change, much as abrupt onsets accompanied by a change in luminance capture attention (e.g., Theeuwes, 1991). Pratt and McAuliffe (2001) showed that both onset and offset peripheral cues presented in isolation result in similar cuing effects at short SOAs, and IOR effects at long SOAs, when target location discrimination is required. However, when onset and offset cues were presented simultaneously, only onset cues facilitated discrimination, suggesting that in cases of simultaneous presentation, onsets dominate capture. Moreover, Boot, Kramer, and Peterson (2005) provided evidence that in an oculomotor capture paradigm, onsets were especially potent at capturing the eyes during goal-directed saccades, but there was no such effect on oculomotor capture by offsets. As a result, they concluded that abruptly onsetting objects receive attentional priority over offsets.

Top-down factors also influence attentional capture. When observers perform an irrelevant feature search task while concurrently performing a demanding secondary memory task, onset capture is diminished (Boot, Brockmole, \& Simons, 2005). Furthermore, Folk and colleagues (Folk \& Remington, 1999; Folk et al., 1992) have provided evidence that onsets capture attention only when observ-

J. D. Cosman, joshua-cosman@uiowa.edu 
ers are set for onset targets. If observers are either cued to or search for a target defined by color rather than by onset status, onsets no longer appear to capture attention (Folk \& Remington, 1999; Folk et al., 1992). However, a recent study by Schreij, Owens, and Theeuwes (2008), using a paradigm nearly identical to that used by Folk et al. (1992), showed that onsets presented simultaneously with the offset of features creating a search array captured attention, despite observers' attentional set for color. This provides further evidence that onsets dominate attentional capture when presented simultaneously with other salient stimuli, even if observers are not set to search for them.

In sum, although some evidence suggests that top-down factors such as attentional set or memory load can affect onset capture, other evidence suggests that onsets are unique in their ability to capture attention in a stimulusdriven manner. In the present article, we ask whether scene complexity affects onset capture; specifically, do abruptly appearing objects capture attention in complex scenes containing many objects? Affirmative and negative answers to this question seem equally plausible. The initial findings on onset capture demonstrated that onset targets produced faster responses than did offset targets across a range of set sizes. If large set sizes correspond to more complex scenes, the initial onset-capture results suggest that scene complexity does not influence attentional capture. However, in classic onset-capture studies, the target can appear as an onset, allowing top-down factors to modulate capture by onsets.

In contrast, other evidence suggests that task-irrelevant targets can be ignored in complex scenes that have a "high perceptual load" (Lavie, 1995). When searching for a target letter among several distractors (high perceptual load displays), observers can disregard an irrelevant flanking letter, but when the target letter is presented by itself (low perceptual load displays), observers cannot disregard the flanker, and the flanker affects reaction times (RTs) to the target (Lavie, 1995; Lavie \& Cox, 1997). Lavie and colleagues have hypothesized that high-load displays are more resource demanding, leaving few attentional resources to "spill over" to the flanker; such spillover can, however, occur in low-load displays. Unfortunately, studies of perceptual load do not shed light on whether onset capture is affected by scene complexity. Targets in perceptual load tasks appear as onsets, which could allow observers to be set for onsets and could prioritize abruptly appearing flankers (as in Eltiti, Wallace, \& Fox, 2005).

To examine the effect of scene complexity - that is, perceptual load - on attentional capture by abrupt onsets, we used a hybrid flanker and feature search task. Observers were presented with a central search array that contained either one (low-load) or six (high-load) letters, all defined as offsets, as shown in Figure 1. Defining the target and distractors as offsets would prevent observers from being set for display-wide onsets (Gibson \& Kelsey, 1998) and should bias attentional control settings toward offsets, not onsets. The search array was flanked above and below by irrelevant distractors, one offset flanker and one onset flanker. If abrupt onsets capture attention in a purely stimulus-driven

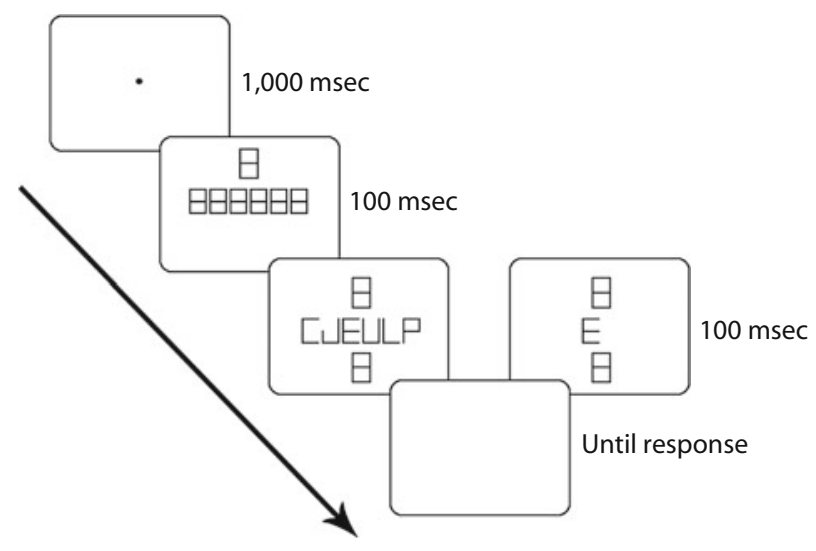

Figure 1. Sequence of events and timing parameters for Experiment 1. Following presentation of a fixation point for $1,000 \mathrm{msec}$, a six-item placeholder array was presented for $100 \mathrm{msec}$, and line segments then disappeared to reveal the search array, which was presented for 100 msec. Search arrays could be high load (six items) or low load (one item). In Experiment 2, timing parameters were the same as in Experiment 1, but in the low-load condition the placeholder array consisted of a single placeholder at the location of the to-be-presented target.

manner, onset flankers should produce flanker interference effects, regardless of the display's perceptual load. Alternatively, if capture by abrupt onsets is reduced in complex, high-load displays, onset distractors should produce flanker interference only in low-load displays.

\section{EXPERIMENT 1}

\section{Method}

Subjects. Twenty University of Iowa undergraduates participated for course credit. All had normal or corrected-to-normal vision.

Stimuli and Procedure. A Macintosh mini-computer displayed stimuli on a 17-in. CRT, and recorded responses and response latencies. The experiment was controlled using MATLAB and the Psychophysics Toolbox (Brainard, 1997).

Observers sat $75 \mathrm{~cm}$ from the monitor in a dimly lit room. We used a task that combined Lavie's (1995) perceptual load paradigm and a typical abrupt onset paradigm (Yantis \& Jonides, 1990). The time course of sample trials appears in Figure 1. A fixation point measuring $0.35^{\circ} \times 0.35^{\circ}$ appeared for $1,000 \mathrm{msec}$, followed by a placeholder array, which appeared for $100 \mathrm{msec}$. In both low- and high-load conditions, the placeholder display contained a central array of six figure-eight placeholders subtending $7.50^{\circ} \times 1.60^{\circ}$ of visual angle, with each placeholder measuring $1.50^{\circ} \times 0.75^{\circ}$, with a distance of $0.45^{\circ}$ between each placeholder. The placeholder for the offset flanker $\left(1.90^{\circ} \times 0.90^{\circ}\right)$ appeared either above or below the six centrally located placeholders. The center of the flanking placeholder was positioned $3.0^{\circ}$ from the fixation point and $4.5^{\circ}$ from the center of the most eccentric placeholders in the central array.

Next, line segments disappeared from the placeholders, including the offset flanker placeholder, to reveal an array of letters constituting the search array. Simultaneously with the offset of the line segments, a letter appeared in a position opposite the offset flanker; this onset flanker had the same measurements as the offset flanker. The resulting high-load display contained two flankers (one offset and one onset) and six centrally located, task-relevant letters containing a target and five distractors. The low-load display, on the other hand, contained two flankers and a single letter (always the target) in one of the six possible locations. This search array remained visible for 
$100 \mathrm{msec}$, so that the time from the placeholder array to the target offset was $200 \mathrm{msec}$, too brief a duration to permit eye movements.

The observers' task was to report the identity of a target letter, which was an $\mathrm{E}$ or an $\mathrm{H}$ either embedded within an array of the five distractor letters U, L, P, C, or J (high load) or presented by itself (low load). Each letter was equally likely to appear in any of the six different positions in the search array. One of the flanker letters was always the letter $\mathrm{S}$, which was neutral with respect to target (and distractor) identities. The other flanker was an $\mathrm{E}$ or an $\mathrm{H}$ congruent or incongruent with the target letter. Observers reported the target's identity by pressing either the " $z$ " or the "/" key, with response keys for the $\mathrm{E}$ and $\mathrm{H}$ targets being counterbalanced between observers. Following 48 practice trials, observers responded to 576 experimental trials. Trials were blocked by load, with each block containing 48 trials of either high- or low-load displays. There were 6 blocks each of high- and low-load displays, resulting in 12 blocks total. Highand low-load blocks were alternated and counterbalanced, such that half of the subjects alternated high, low, high, low, and so on, and the other half of the subjects alternated low, high, low, high, and so on. We told observers to maintain fixation but informed them that the flanker letters, being irrelevant to the task, should be ignored.

With this design, it was possible to examine the effects of taskirrelevant onset and offset flankers on search performance under conditions of both high and low perceptual load. Specifically, the central search array on each trial was flanked by letters that appeared simultaneously both above and below the array. Because the placeholder array preceding the search array contained a placeholder for only one of the two possible flanker locations (either above or below the array), one of the flanker letters always appeared as an onset. On each trial, there was a $50 \%$ chance that the offset flanker would be neutral with respect to the target, leaving a $25 \%$ chance that the onset flanker would be either congruent or incongruent with respect to the target. Onset flankers were equally likely to appear above or below the array. On the basis of the findings of Schreij et al. (2008), onsets would be predicted to dominate capture on each trial, even though observers were set to search for an offset target. Thus, if perceptual load modulates attentional capture by abrupt onsets, we would expect to see capture only in the low-load onset condition.

\section{Results and Discussion}

Across all experiments, RTs of less than $150 \mathrm{msec}$, or greater than 2,000 msec, were excluded from the analyses; this trimming eliminated less than $1 \%$ of the data. Observers' mean RT and error rate data for each condition are shown in Figure 2. We analyzed both accuracy and RT data with a three-factor repeated measures ANOVA, with display load (high vs. low) flanker type (whether the nonneutral flanker appeared as an onset or as an offset), and flanker congruency (congruent vs. incongruent) as factors.

We found a main effect of perceptual load $[F(1,19)=$ $140.2, p<.0001]$, with faster RTs in the low-load condition $(474 \mathrm{msec})$ than in the high-load condition $(598 \mathrm{msec})$. In addition, there was a main effect of onset status $[F(1,19)=$ $6.8, p<.02]$ and flanker congruency $[F(1,19)=10.2$, $p<.005]$. The two-way interactions between load and flanker type $[F(1,19)=0.05$, n.s.], load and flanker congruency $[F(1,19)=1.2$, n.s. $]$, and flanker type and flanker congruency $[F(1,19)=1.3$, n.s. $]$ were not significant, nor was the three-way interaction between load, flanker type, and flanker congruency $[F(1,19)=0.89$, n.s.]. Although planned comparisons revealed a significant flanker effect in the low-load onset condition $(36 \mathrm{msec})[t(19)=2.6$, $p<.01]$, a smaller (19-msec) but significant $[t(19)=2.2$, $p<.05$ ] flanker effect was also observed in the low-load offset condition, which is somewhat anomalous, given our prediction that onset flankers would dominate capture when presented simultaneously with offset flankers.

Error rates did not differ significantly between onset and offset flanker conditions $[F(1,19)=1.9$, n.s. $]$ or between congruent and incongruent flanker conditions $[F(1,19)=2.5$, n.s. $]$. However, there was a significant main effect of load on error rates, with subjects showing higher error rates in the high-load condition $[F(1,19)=$ $12.7, p<.001]$.

Although these results indicate that onset capture is affected by perceptual load, the finding of capture by both onset and offset flankers does not fit well with previous studies. On the one hand, the contingent involuntary orienting hypothesis of Folk et al. (1992) would pre-

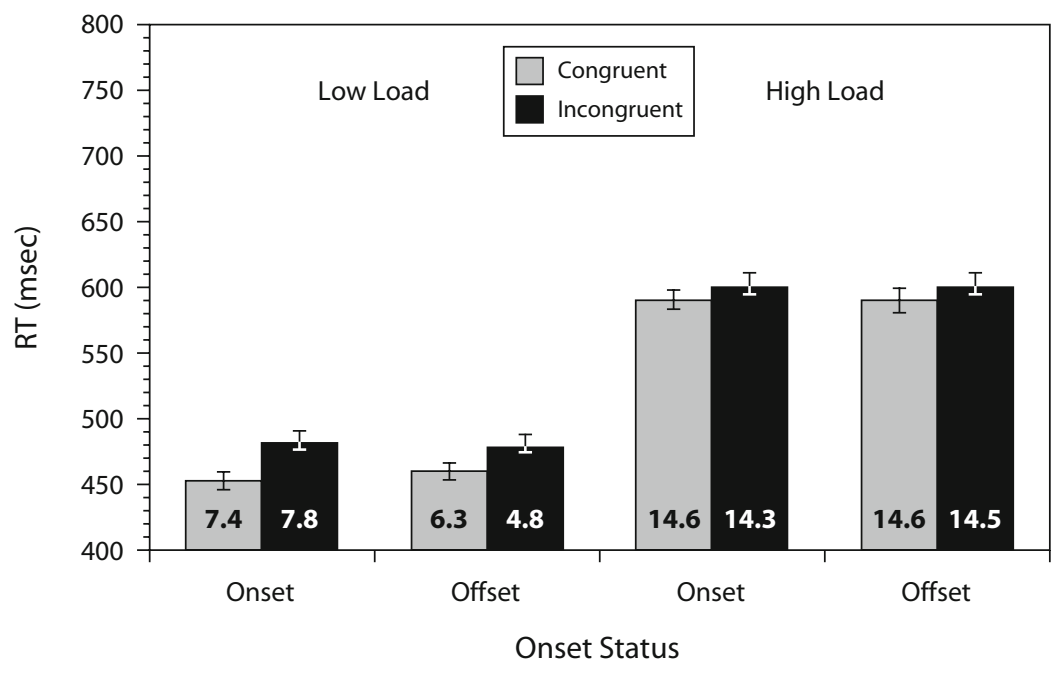

Figure 2. Mean reaction times for high- and low-load conditions when flankers were onsets or offsets in Experiment 1. Error rates for each condition are indicated in white at the base of the graph. Error bars represent $95 \%$ within-subjects confidence intervals. 
dict capture only when the flanker was an offset, because observers were set to search for an offset target. On the other hand, the results of Schreij et al. (2008) would predict capture only when the flanker was an onset, because their findings showed that onsets presented simultaneously with a search array dominate capture, regardless of attentional set.

One reason we might have observed attentional capture in the low-load offset condition is because observers were presented with a placeholder array containing six offsetting placeholders, thereby generating a large offset transient. As a result, it is possible that this offset transient set display-wide attentional control settings (see Gibson \& Kelsey, 1998) and caused offset flankers to capture attention, driving the flanker effect seen in the low-load offset condition. We tested this hypothesis in Experiment 2 with a paradigm identical to that used in Experiment 1, with the exception that a single placeholder preceded the target in the low-load condition (Figure 1). If the offset transient produced by the large placeholder array in Experiment 1 was responsible for the offset capture we observed in the low-load condition of Experiment 1, we would expect the results of Experiment 2 to show capture only in the low-load onset condition.

\section{EXPERIMENT 2}

\section{Method}

Subjects. Twenty University of Iowa undergraduates participated in a single session for course credit. All observers had normal or corrected-to-normal vision.

Stimuli and Procedure. The design of Experiment 2 was identical to that of Experiment 1, except that in the low-load condition the search array was preceded by a single placeholder at the location of the target. This design eliminated the large offset transient present in Experiment 1.

\section{Results and Discussion}

Mean RT and error rate data for each condition are shown in Figure 3. As in Experiment 1, both accuracy and RT data were analyzed with a three-factor repeated measures ANOVA, with display load (high vs. low) onset status (onset vs. offset), and flanker congruency (congruent vs. incongruent) as factors.

We observed a main effect of load $[F(1,19)=65.7$, $p<.0001]$, where RTs in the low-load condition were faster overall $(497 \mathrm{msec})$ than those in the high-load condition $(667 \mathrm{msec})$. In addition, there was a main effect of congruency $[F(1,19)=7.5, p<.02]$, driven by the significant flanker effect in the low-load onset condition. There was no main effect of onset status $[F(1,19)=$ 2.0, n.s.], and the two-way interactions between load and flanker type $[F(1,19)=.05$, n.s.], load and flanker congruency $[F(1,19)=1.2$, n.s. $]$, and flanker type and flanker congruency $[F(1,19)=1.3$, n.s.] were not significant. However, the three-way interaction of load, onset status, and congruency was significant $[F(1,19)=$ $5.8, p<.03$ ], indicating that onset capture depended on the level of perceptual load. Most important, Figure 3 reveals that attentional capture by the irrelevant distractors was observed only in the low-load onset condition, as confirmed with planned comparisons on the compatible versus incompatible conditions for each of the four onset-by-load conditions. There was a significant flanker effect in the low-load onset condition only $(30 \mathrm{msec})$ $[t(19)=3.8, p<.001]$; none of the other flanker effects were significant $(t \mathrm{~s}<1.3, p \mathrm{~s}>.30)$. Thus, it appears that the offset transient produced by the placeholder array in Experiment 1 was responsible for the significant flanker effect in the low-load offset condition.

Error rates did not differ significantly between onset and offset flanker conditions $[F(1,19)=1.3$, n.s. $]$ or between congruent and incongruent flanker conditions

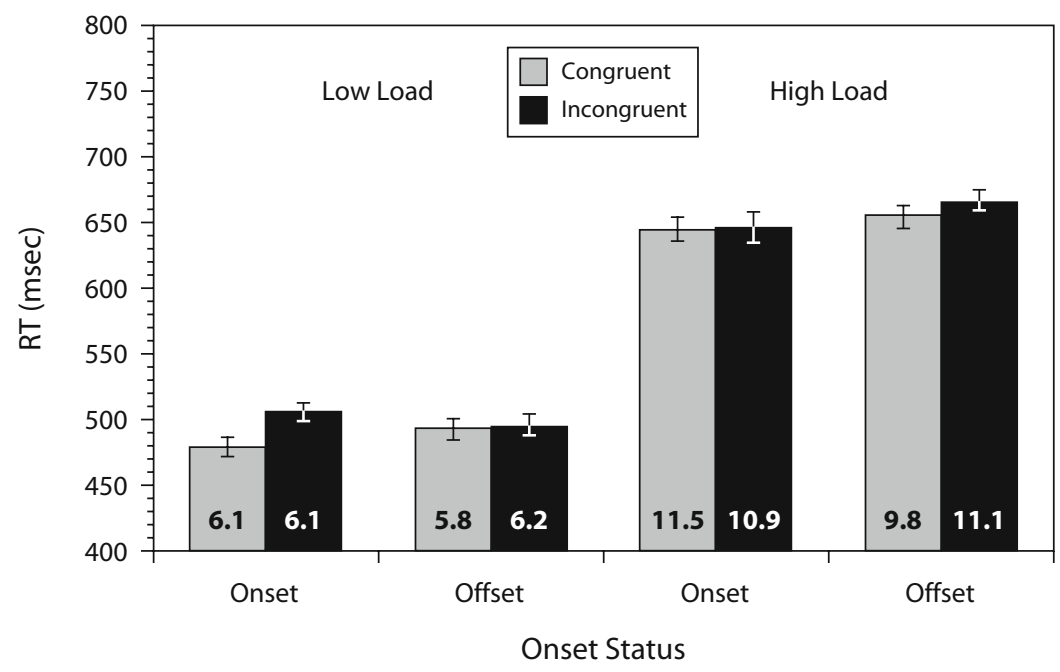

Figure 3. Mean reaction times for high- and low-load conditions when flankers were onsets or offsets in Experiment 2. Error rates for each condition are indicated in white at the base of the graph. Error bars represent $95 \%$ within-subjects confidence intervals. 
$[F(1,19)=1.1$, n.s.]. However, there was a significant main effect of load on error rates, with subjects showing higher error rates in the high-load condition $[F(1,19)=$ $10.2, p<.01]$.

These results indicate that onset capture is affected by perceptual load. When observers searched through complex, high-load displays, they were not captured by abruptly appearing irrelevant distractors. However, onset capture was robust when observers viewed displays in which the target appeared alone and required relatively little attentional processing (or resources, as proposed by Lavie, 1995). Thus, available attentional resources may modulate attentional capture. When attentional resources are abundant in the low-load condition, observers are susceptible to capture by irrelevant information because of spillover to the flankers; when attentional processes are exhausted by a demanding search in the high-load condition, there is no spillover, and no flanker effects emerge.

Although the results of Experiment 2 indicate that perceptual load modulates onset capture, it is possible that capture by abrupt onsets is masked by long RTs in the high-load condition. Specifically, generalized slowing in the high-load condition may have obscured any evidence of onset capture. To rule out this possibility, we manipulated task difficulty independently of perceptual load in Experiment 3. Observers viewed arrays that contained a single target letter and two irrelevant letters, as in the low-load condition of Experiment 2. The target letter appeared as either a black letter on a white background (the high-contrast condition, which was a replication of the low-load condition in Experiment 2) or as a light gray letter on a white background (the low-contrast condition). This contrast manipulation allowed us to vary task difficulty without increasing the perceptual load of the display (also see Lavie \& Cox, 1997). If the results of Experiment 2 merely reflect a task difficulty or gen- eralized slowing effect, we would expect to see onset capture in the high-contrast condition only. However, if the results of Experiment 2 were due to perceptual load, we would expect to see onset capture in both high- and low-contrast conditions because both of these conditions presented only a single target and thus involved a low perceptual load.

\section{EXPERIMENT 3}

\section{Method}

Subjects. Twenty University of Iowa undergraduates participated in a single session for course credit. All observers had normal or corrected-to-normal vision.

Stimuli and Procedure. For both conditions, displays were identical to the low-load display in Experiment 2, with the following exception: To make the task more difficult without increasing perceptual load, the contrast of the target in the low-contrast condition was decreased by $50 \%$, as compared with the target letter in the high-contrast condition

\section{Results and Discussion}

Mean RT and error rate data for each condition are shown in Figure 4. Both accuracy and RT data were analyzed with a three-factor repeated measures ANOVA, with target contrast (high vs. low) onset status (onset vs. offset), and flanker congruency (congruent vs. incongruent) as factors.

We observed a main effect of target contrast $[F(1,19)=$ 16.92, $p<.001]$, with longer RTs in the low-contrast condition $(623 \mathrm{msec})$ than in the high-contrast condition $(518 \mathrm{msec})$. No other main effects or interactions approached significance $\left(F_{\mathrm{S}}<2.2, p \mathrm{~s}>.11\right)$. Most important, planned comparisons revealed that attentional capture was observed in both the low- and high-contrast onset conditions: There were significant flanker effects in the low-contrast onset condition $(38 \mathrm{msec})[t(19)=2.9$,

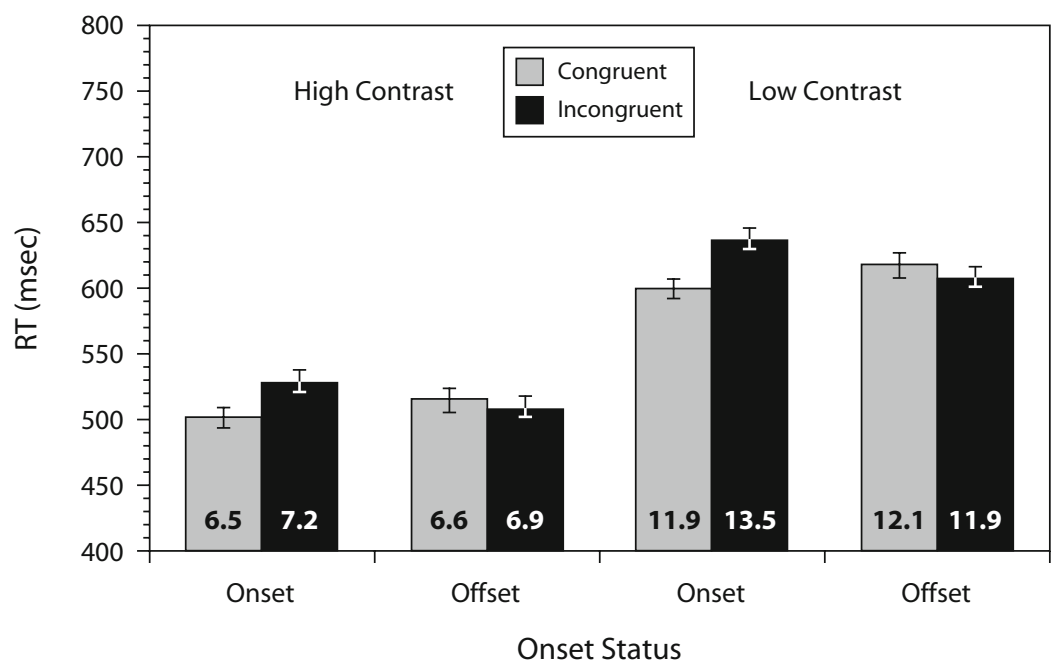

Figure 4. Mean reaction times for high-contrast (easy search) and low-contrast (difficult search) target conditions in Experiment 3. Error rates for each condition are indicated in white at the base of the graph. Error bars represent $95 \%$ within-subjects confidence intervals. 
$p<.01]$ and the high-contrast onset condition $(27 \mathrm{msec})$ $[t(19)=2.9, p<.01]$.

Error rates did not differ significantly between onset and offset flanker conditions $[F(1,19)=0.9$, n.s. $]$ or between congruent and incongruent flanker conditions $[F(1,19)=0.7$, n.s. $]$. However, there was a significant main effect of target contrast on error rates, with subjects showing higher error rates in the low-contrast condition $[F(1,19)=7.6, p<.02]$.

These results indicate that the effect observed in Experiment 2 was not due to the difficulty of our search task, since we observed onset capture even in a relatively difficult low-contrast condition. This further suggests that increasing perceptual load per se can diminish onset capture by task-irrelevant objects.

\section{GENERAL DISCUSSION}

Across three experiments, we demonstrated that the ability of an abruptly appearing object to capture attention depends on perceptual load. Our findings contrast with recent reports showing that abruptly appearing objects capture attention in a mandatory, stimulus-driven manner (Christ \& Abrams, 2006; Neo \& Chua, 2006; Schreij et al., 2008). Our novel findings imply that onset capture may not occur in all displays but, instead, may depend on scene complexity. Furthermore, the lack of capture by offset flankers in Experiments 2 and 3, despite an attentional set for offsets, is inconsistent with the claim that attention capture is contingent solely on top-down control settings (Folk et al., 1992).

There are several potential interpretations of our findings. For example, load theory (Lavie, 1995; Lavie \& Tsal, 1994) would suggest that, as perceptual resources are exhausted, attention becomes less able to be captured by abrupt, irrelevant flankers. However, the lack of capture by offset flankers in Experiments 2 and 3 is inconsistent with a strong version of load theory, which predicts that load only, not onset/offset status, will affect RTs. There are also nonresource accounts that could explain our results. For example, attention may be more narrowly focused when targets in high-load displays are searched for, minimizing onset capture, consistent with previous claims (Yantis \& Johnston, 1990; but see Christ \& Abrams, 2006). Irrespective of the exact mechanism that allows perceptual load to modulate onset capture, our present results are theoretically important in providing the first demonstration that perceptual load indeed modulates attentional capture by abrupt onsets.

The interpretation that onset capture is not purely stimulus driven is in line with Boot et al.'s (2005) recent finding that abruptly appearing objects are less likely to capture attention under conditions of high cognitive load, when central processing stages are occupied by a concurrent working memory task. Further support for a modulation of onset capture comes from Santangelo, Finoia, Raffone, Belardinelli, and Spence (2008), who demonstrated that increasing perceptual load in a spatial cuing task reduces a cue's ability to attract attention. In high-load situations, valid exogenous spatial precues did not result in the RT benefit typically seen in such tasks, indicating that perceptual load influenced the ability of the abruptly appearing precues to capture attention.

Our finding of offset capture in the low-load condition of Experiment 1 deserves mention. The offset capture observed in Experiment 1 suggests that contingent attentional capture might be modulated by perceptual load. Assuming that offset capture was due to contingent capture established by the large offset transient in this experiment, we observed contingent capture in the low-load condition only. We are tentative in drawing this conclusion, because the present experiments did not systematically examine contingent capture. We plan to study this issue further.

Although our results suggest that scene complexity may modulate capture by abruptly appearing objects, there is evidence for capture by onsets in complex, rendered scenes (Brockmole \& Henderson, 2008). In their task, Brockmole and Henderson presented participants with a realistically rendered real-world scene in which one of the objects onsets abruptly on half of the trials. Observers were more likely to fixate objects that onset abruptly, suggesting that these objects captured attention despite the high information content of the scene. There seem to be several explanations for the apparent discrepancy between our results and Brockmole and Henderson's findings. For example, real-world scenes have semantic consistency that might reduce perceptual load, even though the scene contains several objects. Also, all of the items in our search array were relevant to the task observers performed. Capture may not depend on the complexity of the scene per se, but rather on the amount of relevant perceptual information being processed at the time of the onset. In short, there may be many factors, including perceptual load, that affect capture by abrupt onsets.

\section{AUTHOR NOTE}

This research was supported in part by a grant from the National Science Foundation (BCS 03-39171), awarded to S.P.V. We thank Jay Pratt, Angus Gellatly, and Daryl Wilson for helpful comments on an earlier version of this article. We also thank Nicholas Gaspelin and Vanessa Jimenez for assistance collecting data. Correspondence should be addressed to J. D. Cosman, Department of Psychology, University of Iowa, E11 Seashore Hall, Iowa City, IA 52242-1407 (e-mail: joshua-cosman@ uiowa.edu).

\section{REFERENCES}

Abrams, R. A., \& Christ, S. E. (2003). Motion onset captures attention. Psychological Science, 14, 427-432.

Boot, W. R., Brockmole, J. R., \& Simons, D. J. (2005). Attention capture is modulated in dual-task situations. Psychonomic Bulletin \& Review, 12, 662-668.

Boot, W. R., Kramer, A. F., \& Peterson, M. S. (2005). Oculomotor consequences of abrupt object onsets and offsets: Onsets dominate oculomotor capture. Perception \& Psychophysics, 67, 910-928.

Brainard, D. H. (1997). The Psychophysics Toolbox. Spatial Vision, 10, 433-436.

Brockmole, J. R., \& Henderson, J. M. (2008). Prioritizing new objects for eye fixation in real-world scenes: Effects of object-scene consistency. Visual Cognition, 16, 375-390.

Christ, S. E., \& Abrams, R. A. (2006). Abrupt onsets cannot be ignored. Psychonomic Bulletin \& Review, 13, 875-880.

Eltiti, S., Wallace, D., \& Fox, E. (2005). Selective target processing: 
Perceptual load or distractor salience? Perception \& Psychophysics, 67, 867-885.

Folk, C., \& Remington, R. W. (1999). Can new objects override attentional control settings? Perception \& Psychophysics, 61, 727-739.

Folk, C., Remington, R. W., \& Johnston, J. C. (1992). Involuntary covert orienting is contingent on attentional control settings. Journal of Experimental Psychology: Human Perception \& Performance, 18, 1030-1044.

Franconeri, S. L., \& Simons, D. J. (2003). Moving and looming stimuli capture attention. Perception \& Psychophysics, 65, 999-1010.

Franconeri, S. L., Simons, D. J., \& Junge, J. A. (2004). Searching for stimulus-driven shifts of attention. Psychonomic Bulletin \& Review, 11, 876-881.

Gibson, B. S., \& Kelsey, E. M. (1998). Stimulus-driven attentional capture is contingent on attentional set for displaywide visual features. Journal of Experimental Psychology: Human Perception \& Performance, 24, 699-706.

Lavie, N. (1995). Perceptual load as a necessary condition for selective attention. Journal of Experimental Psychology: Human Perception \& Performance, 21, 451-468.

Lavie, N. \& Cox, S. (1997). On the efficiency of visual selective attention: Efficient visual search results in inefficient distractor rejection. Psychological Science, 8, 395-396.

Lavie, N., \& Tsal, Y. (1994). Perceptual load as a major determinant of the locus of selection in visual attention. Perception \& Psychophysics, 56, 183-197.
Neo, G., \& ChuA, F. (2006). Capturing focused attention. Perception \& Psychophysics, 68, 1286-1296.

Pratt, J., \& McAuliffe, J. (2001). The effects of onsets and offsets on visual attention. Psychological Research, 65, 185-191.

Santangelo, V., Finoia, P., Raffone, A., Belardinelli, M. O., \& SPENCE, C. (2008). Perceptual load affects exogenous spatial orienting while working memory load does not. Experimental Brain Research, 134, 371-382.

Schreis, D., Owens, C., \& Theeuwes, J. (2008). Abrupt onsets capture attention independent of top-down control settings. Perception \& Psychophysics, 70, 208-218.

THEEUWES, J. (1991). Exogenous and endogenous control of attention: The effect of visual onsets and offsets. Perception \& Psychophysics, 49, 83-90.

YANTis, S., \& Johnston, J. C. (1990). On the locus of visual selection: Evidence from focused attention tasks. Journal of Experimental Psychology: Human Perception \& Performance, 16, 135-149.

Yantis, S., \& Jonides, J. (1984). Abrupt visual onsets and selective attention: Evidence from visual search. Journal of Experimental Psychology: Human Perception \& Performance, 10, 601-621.

Yantis, S., \& Jonides, J. (1990). Abrupt visual onsets and selective attention: Voluntary versus automatic allocation. Journal of Experimental Psychology: Human Perception \& Performance, 16, 121-134.

(Manuscript received March 9, 2008;

revision accepted for publication November 12, 2008.) 